\title{
Isolation and Identification of Actinomycetes Strains from Switzerland and their Biotechnological Potential
}

\author{
Fabienne Arn§, David Frasson ${ }^{\mathrm{b}}$, Ivana Kroslakovab ${ }^{\mathrm{a}}$, Fabio Rezzonicoc ${ }^{\mathrm{c}}$, Joël F. Pothierc, \\ Rainer Riedla ${ }^{a}$ and Martin Sievers ${ }^{\mathrm{b} *}$
}

\begin{abstract}
Actinomycetes strains isolated from different habitats in Switzerland were investigated for production of antibacterial and antitumoral compounds. Based on partial 16S rRNA gene sequences, the isolated strains were identified to genus level. Streptomyces as the largest genus of Actinobacteria was isolated the most frequently. A screening assay using the OmniLog instrument was established to facilitate the detection of active compounds from actinomycetes. Extracts prepared from the cultivated strains able to inhibit Staphylococcus aureus and Escherichia coli were further analysed by HPLC and MALDI-TOF MS to identify the produced antibiotics. In this study, the bioactive compound echinomycin was identified from two isolated Streptomyces strains. Natural compounds similar to TPU-0037-C, azalomycin $\mathrm{F}_{4 \mathrm{a}}$ 2-ethylpentyl ester, a derivative of bafilomycin A1, milbemycin- $\alpha 8$ and dihydropicromycin were detected from different isolated Streptomyces strains. Milbemycin- $\alpha 8$ showed cytotoxic activity against HT-29 colon cancer cells. The rare actinomycete, Micromonospora sp. Stup16_ C148 produced a compound that matches with the antibiotic bottromycin A2. The draft genome sequence from Actinokineospora strain B136.1 was determined using Illumina and nanopore-based technologies. The isolated strain was not able to produce antibacterial compounds under standard cultivation conditions. The antiSMASH bioinformatics analyses of the genome from strain B136.1 identified biosynthetic gene clusters with identity values between $4 \%$ to $90 \%$ to known gene clusters encoding antibiotics. The combinations of cultivation conditions, screening assays, analytical methods and genome mining are important tools to characterize strains of actinomycetes for the identification of their potential to produce natural compounds with antimicrobial activity.
\end{abstract}

Keywords: Actinomycetes · Antibacterial compounds · AntiSMASH · Cultivation · Drug discovery

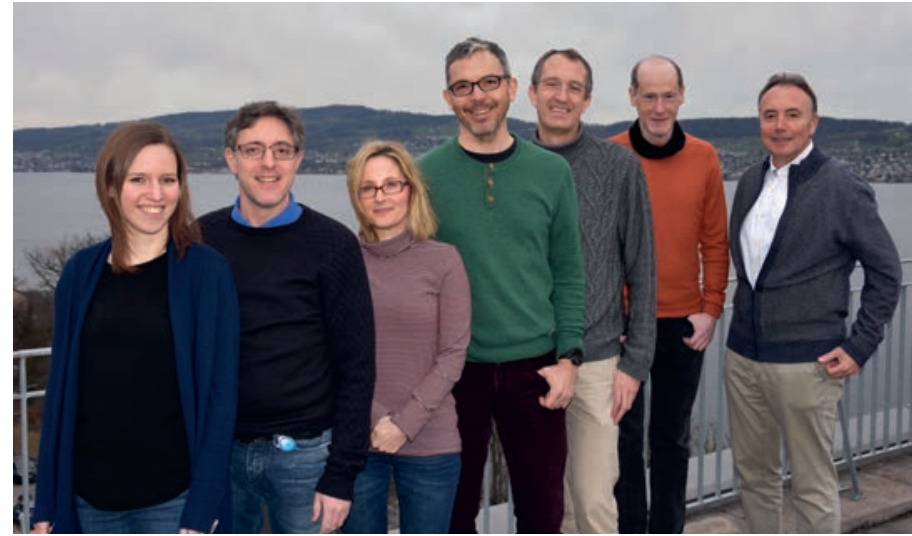

From left to right: Fabienne Arn, David Frasson, Ivana Kroslakova, Fabio Rezzonico, Joël F. Pothier, Rainer Riedl and Martin Sievers

\section{Introduction}

The development of antibiotic resistance is a major global threat for public health. The past decades have seen a dramatic increase in bacteria that are resistant to one or multiple antibiotics. Unfortunately, the industry pipelines for novel antibiotics have run dry. Therefore, there is tremendous need for the generation of new strategies to fight infections caused by resistant bacterial pathogens. ${ }^{[1]}$ Besides the design and synthesis approach toward novel antibiotics, microbial extract screening has been and will be a valuable source of extraordinary molecules with antibiotic activity. ${ }^{[2]}$

Actinomycetes are a diverse group of Gram-positive bacteria, which are able to form spores and are characterized by a filamentous and branching growth pattern. Spore germination results in a vegetative growth pattern of actinomycetes forming substrate mycelium. ${ }^{[3]}$ The transition from vegetative growth to the formation of reproductive aerial hyphae is the response of the cells to nutrient depletion. A proportion of the vegetative mycelium lyses to support the remaining cells with nutrition. An increased production of secondary metabolites is observed during this development stage. The antibiotics produced during this development stage inhibit other microorganisms competing for the same nutrition. ${ }^{[4]}$ Spore formation, pigment production and antibiotic production are regulated by quorum sensing. The autoinducer $\gamma$-butyrolactone system seems to be limited to the phylum Actinobacteria and plays important roles as signalling molecule. ${ }^{[5]}$ Most of the currently used antibiotics derive from actinomycetes with Streptomyces being the main producer. One of the first antibiotics discovered was streptomycin obtained from Streptomyces griseus which has been isolated from the farm soil of the Rutgers Agriculture School and was used to treat tuberculosis. For this work, Selman Waksman received the Nobel Prize in $1952 .{ }^{[6]}$ To honour the contribution of 
S. griseus to human healthcare, the bacterium is now officially recognised as New Jersey's State Microbe (The Scientist, May 13, 2019). Most antibiotics used today were discovered during the period 1940-1960, which is referred to as the golden era of antibiotic discovery. ${ }^{[7]}$ The antibiotics produced from actinomycetes belong to different chemical classes such as aminoglycosides (streptomycin, gentamycin, neomycin, tobramycin), glycopeptides (vancomycin, teicoplanin), macrolides (erythromycin, carbomycin), amphenicols (chloramphenicol), ansamycins (rifamycin), tetracyclines (oxytetracycline, chlortetracycline), lincosamides (clindamycin), lipopeptides (daptomycin), $\beta$-lactams (cephalosporin), and aminocoumarins (novobiocin). ${ }^{[8]}$ Actinomycetes represent a promising source to isolate novel antibacterial compounds, which are urgently needed to combat antibiotic-resistant bacteria. Improved screening methods led to the discovery of the cyclic peptide compound lassomycin from Lentzea kentuckyensis which is a Clp protease inhibitor and active against drug-resistant strains of Mycobacterium tuberculosis. ${ }^{[9]}$ The majority of the compounds with biological activities produced from actinomycetes is still undiscovered. In the present study, Streptomyces and rare actinomycetes strains isolated in Switzerland were investigated for their antibacterial and cytotoxic activities.

\section{Isolation and Identification of Strains of Actinobacteria from Switzerland}

Nine strains of actinomycetes collected from different habitats in Switzerland were investigated for antibacterial and cytotoxic activities (Table 1). For isolation of actinomycetes strains from soil, the collected samples were air dried in an incubator at $28{ }^{\circ} \mathrm{C}$ for seven days. Then $10 \mathrm{~g}$ of dried soil was resuspend- ed in $100 \mathrm{ml}$ sterile deionized water for 30 mins under shaking conditions at $28{ }^{\circ} \mathrm{C}$. Serial dilutions of the supernatants of the obtained homogenates were plated on different agar media. One medium used for cultivation of isolates from environmental samples was GYM4, composed of $4 \mathrm{~g}$ each of glucose, yeast extract and malt extract per litre of demineralized water. ${ }^{[10]}$ In addition, media from the International Streptomyces Project described by Shirling and Gottlieb ${ }^{[11]}$ and ZSSE agar containing starch and soil extract ${ }^{[12]}$ were used. Suspension of $100 \mathrm{mg} / \mathrm{L}$ nystatin, 100 $\mathrm{mg} / \mathrm{L}$ cycloheximide and $50 \mathrm{mg} / \mathrm{L}$ nalidixic acid were added to the media after autoclaving to prevent growth of fungi and Gramnegative bacteria. Inoculated agar plates were incubated at $28{ }^{\circ} \mathrm{C}$ for up to one month. Obtained colonies were further purified on R2A agar (Sigma Aldrich). Pure cultures were obtained by repeated streaking on R2A agar. The isolated strains showed different morphological appearance with respect to colony shape and size on agar plate and some isolates identified as members of the genus Micromonospora were able to produce red pigments. The isolated strains were maintained as glycerol stocks in cryocultures at $-80{ }^{\circ} \mathrm{C}$. Determination of partial $16 \mathrm{~S}$ rRNA gene sequences and BLASTn analyses classified the investigated actinomycetes strains into three different genera. The 16S rRNA gene was amplified from the isolated DNA of the different strains using primers $8 f$ (5'- AGA GTT TGA TCC TGG CTC AG -3') and 1392r (5' - ACG GGC GGT GTG TAC A -3').[13] A 1,384 bp fragment spanning from the V1 to part of the V9 region of the 16S rRNA gene was obtained and compared with available sequences in the GenBank/EMBL/DDBJ database using BLASTn search, revealing an identity to genus level. The identified genera were Streptomyces (7 isolates), Micromonospora (1 isolate) and Actinokineospora (1 isolate).

Table 1. Investigated strains for production of antibacterial and cytotoxic compounds.

\begin{tabular}{|c|c|c|c|c|c|}
\hline Genus & Origin & Coordinates & Inhibition & {$[m / z]$} & Compound \\
\hline \multicolumn{6}{|l|}{ Strain } \\
\hline $\begin{array}{l}\text { Streptomyces } \\
\text { Stup16_B49.2 }\end{array}$ & Grassland soil & $\begin{array}{l}47^{\circ} 13^{\prime} 59.0^{\prime \prime} \mathrm{N} \\
8^{\circ} 07^{\prime} 54.6^{\prime \prime} \mathrm{E}\end{array}$ & $\begin{array}{l}\text { S. aureus } \\
\text { E. coli }\end{array}$ & $\begin{array}{l}1123.403 \\
{[\mathrm{M}+\mathrm{Na}]^{+}}\end{array}$ & Echinomycin $^{\mathrm{a}}$ \\
\hline $\begin{array}{l}\text { Streptomyces } \\
\text { sima1_6 }\end{array}$ & Moor & $\begin{array}{l}47^{\circ} 14^{\prime} 43.0^{\prime \prime} \mathrm{N} \\
8^{\circ} 30^{\prime} 26.4^{\prime \prime} \mathrm{E}\end{array}$ & S. aureus & $\begin{array}{l}1123.403 \\
{[\mathrm{M}+\mathrm{Na}]^{+}}\end{array}$ & Echinomycin $^{\mathrm{a}}$ \\
\hline $\begin{array}{l}\text { Streptomyces } \\
\text { Stup16_B146 }\end{array}$ & Grassland soil & $\begin{array}{l}47^{\circ} 13 ' 59.0^{\prime \prime} \mathrm{N} \\
8^{\circ} 07^{\prime} 54.6^{\prime \prime} \mathrm{E}\end{array}$ & S. aureus & $\begin{array}{l}825.522 \\
{[\mathrm{M}+\mathrm{H}]^{+}}\end{array}$ & TPU-0037-C \\
\hline $\begin{array}{l}\text { Streptomyces } \\
\text { OPOK_MB_B11 }\end{array}$ & Soil & $\begin{array}{l}47^{\circ} 13^{\prime} 05.5^{\prime \prime} \mathrm{N} \\
8^{\circ} 40^{\prime} 50.0^{\prime \prime} \mathrm{E}\end{array}$ & S. aureus & $\begin{array}{l}1202.751 \\
{[\mathrm{M}+\mathrm{Na}]^{+}}\end{array}$ & $\begin{array}{l}\text { Azalomycin } \mathrm{F}_{4 \mathrm{a}} \\
\text { 2-ethylpentyl ester }\end{array}$ \\
\hline $\begin{array}{l}\text { Streptomyces } \\
\text { Stup18_J70 }\end{array}$ & Forest soil & $\begin{array}{l}46^{\circ} 20^{\prime} 41.0^{\prime \prime} \mathrm{N} \\
8^{\circ} 00^{\prime} 27.4^{\prime \prime} \mathrm{E}\end{array}$ & S. aureus & $\begin{array}{l}787.449 \\
{[\mathrm{M}+\mathrm{Na}]^{+}}\end{array}$ & $\begin{array}{l}\text { Bafilomycin A1 } \\
\text { derivative }\end{array}$ \\
\hline $\begin{array}{l}\text { Streptomyces } \\
\text { OPOK_MB_A9 }\end{array}$ & Soil & $\begin{array}{l}47^{\circ} 13^{\prime} 06.6^{\prime \prime} \mathrm{N} \\
8^{\circ} 41^{\prime} 18.5^{\prime \prime} \mathrm{E}\end{array}$ & HT-29 & $\begin{array}{l}701.444 \\
{[\mathrm{M}+\mathrm{H}]^{+}}\end{array}$ & Milbemycin- $\alpha 8$ \\
\hline $\begin{array}{l}\text { Micromonospora } \\
\text { Stup16_C148 }\end{array}$ & $\begin{array}{l}\text { Soil of a } \\
\text { riverbank }\end{array}$ & $\begin{array}{l}47^{\circ} 24^{\prime} 35.1^{\prime \prime} \mathrm{N} \\
8^{\circ} 26^{\prime} 58.9^{\prime \prime} \mathrm{E}\end{array}$ & S. aureus & $\begin{array}{l}823.440 \\
{[\mathrm{M}+\mathrm{H}]^{+}}\end{array}$ & Bottromycin A2 \\
\hline $\begin{array}{l}\text { Streptomyces } \\
\text { W367A }\end{array}$ & Worm castings & $\begin{array}{l}47^{\circ} 08^{\prime} 04.1^{\prime \prime} \mathrm{N} \\
8^{\circ} 07^{\prime} 56.5^{\prime \prime} \mathrm{E}\end{array}$ & S. aureus & $\begin{array}{l}550.343 \\
{[\mathrm{M}+\mathrm{Na}]^{+}}\end{array}$ & Dihydropicromycin \\
\hline $\begin{array}{l}\text { Actinokineospora } \\
\text { B136.1 }\end{array}$ & Grassland soil & $\begin{array}{l}47^{\circ} 13^{\prime} 59.0^{\prime \prime} \mathrm{N} \\
8^{\circ} 07^{\prime} 54.6^{\prime \prime} \mathrm{E}\end{array}$ & Genome mining & & \\
\hline
\end{tabular}




\section{Primary Screening of the Isolated Strains for Antibacterial Activities}

The isolated actinomycetes strains were tested for their ability to produce antibacterial compounds against Staphylococcus aureus ATCC 25923 and Escherichia coli TOP10F' (Thermo Fisher Scientific) using the cross-streak method. This preliminary screening is a simple and rapid method to investigate strains for production of antimicrobial metabolites. Three different media, R2A, GYM4 and NL148sb, were used for the cross-streaking experiment. NL148sb was described recently by Lütz and coworkers. ${ }^{[14]}$ An isolated strain of Actinobacteria was cross-streaked as a single central line vertical on each of the three agar plates and incubated for 5 to 7 days at $28^{\circ} \mathrm{C}$ until growth occurred. E. coli and $S$. aureus as test strains were each cross-streaked horizontally as two separate lines left and right from the original streak of the Actinobacteria strain. The plates were incubated for $24 \mathrm{~h}$ at $37^{\circ} \mathrm{C}$ to determine the growth of the tested strains. Antagonism is observed by the inhibition of growth of selected pathogenic bacteria used as test strains. Inhibition zones were estimated qualitatively, whereby a strong inhibition was indicated by growth inhibition of the line of the tested strain at least by two-third of its length. Weak inhibition was indicated by attenuated growth of the test strain close to the area of the actinomycetes strain used in the assay. Of nine strains of actinomycetes tested for antibacterial and antitumoral compound production, seven strains showed strong inhibition against $S$. aureus and weak to no inhibition against $E$. coli in the preliminary screening, and one strain was not able to inhibit the two test strains using the three different media in the cross-streaking method. One strain showed cytotoxic activity against the human colon cancer cell line HT-29.

The following seven strains which showed antibacterial activity using the cross-streaking method are: Streptomyces sp. strain Stup16_B146, Streptomyces sp. strain Stup16_B49.2 (the two strains were isolated from different grassland soils), Streptomyces sp. strain OPOK_MB_B11 (isolated from soil), Streptomyces sp. strain sima 1_6 (isolated from moor), Streptomyces sp. strain Stup18_J70 (isolated from forest soil), Streptomyces sp. strain W367A (isolated from worm castings) and Micromonospora sp. strain Stup16_C148 (isolated from soil of a riverbank). These seven isolates, which showed antibacterial activity, were selected for further analysis to identify the antibacterial compounds.

\section{Secondary Screening of Compounds for Antibacterial Activities Using the OmniLog Instrument}

A colorimetric assay in combination with respiratory growth curves was applied to optimise the screening method for determination of antibacterial activities from extracted compounds. The OmniLog instrument (Biolog) was used to measure growth inhibition of $E$. coli TOP $10 F^{\prime}$ and $S$. aureus ATCC 25923. For direct visualisation of inhibition, microtiter plate wells were inoculated with $10^{3}$ bacterial cells/ml of each strain in TSB broth and incubated in the presence of the compound. The compound to be investigated for inhibition was dissolved in DMSO, either in form of the extract generated from the supernatant or mycelium of the cultivated strain, or as fraction obtained from HPLC. The used dyes were Biolog Redox Dye Mix A for E. coli and Biolog Redox Dye Mix H for S. aureus. Chloramphenicol $(37 \mu \mathrm{M})$ and kanamy$\operatorname{cin}(58 \mu \mathrm{M})$ were used as positive controls and DMSO 5.3\% $(v / v)$ as negative control. Kinetics of bacterial growth and dye reduction were monitored with a camera of the OmniLog instrument continuously over the course of the experiment up to $24 \mathrm{~h}$. Inhibition of bacterial growth does not change the colour of the included dye and no visible metabolic activity of the included strains ( $E$. coli or $S$. aureus) is thus detected. Reduction of the dyes indicates growth of the test strains used in the assay. By comparing both the colour of the used dye and kinetic growth curves within each well, the antibacterial effect of the investigated compound was measured.
A combination of dye reduction and visualisation of respiratory growth curves provides a reliable and highly informative method to determine the antibacterial activity of investigated compounds.

\section{Preparation of Extracts and HPLC Analysis}

Culture supernatants from actinomycetes strains, which showed inhibition in the cross-streaking experiment, were extracted with ethyl acetate from culture grown in all three media (GYM4, R2A and NL148sb) for 7 days with shaking at $100 \mathrm{rpm}$ at $28{ }^{\circ} \mathrm{C}$. The extracts were evaporated to dryness and dissolved in methanol. Streptomyces sp. strain sima1_6 showed potential antibacterial activity in the preliminary cross-streaking assay, but the supernatant of the cultivated strain failed to inhibit both $E$. coli and S. aureus. For isolation of the antibacterial compound from strain sima1_6 grown on GYM4 agar, the fragmented mycelium and agar was extracted overnight with acetone, concentrated on a rotary evaporator and dissolved in methanol. The methanol solvents containing the extracts were subjected to HPLC (HPLC Series 1200, Agilent Technologies) analysis. The extracts were chromatographed through a precolumn (Strategy C18-2) followed by the HPLC column (Uptisphere Strategy C18-HQ), both from Interchim. The column temperature was $40{ }^{\circ} \mathrm{C}$. Elution was performed using a flow-rate of $1 \mathrm{ml} / \mathrm{min}$. Elution solvent changed from $95 \%$ water, $4.8 \%$ methanol and $0.2 \%$ acetic acid (solvent $\mathrm{A}$ ) to $95 \%$ methanol, $4.8 \%$ water and $0.2 \%$ acetic acid (solvent B) in 10 mins, followed by additional 8 mins with solvent B. Single fractions were collected, concentrated and dissolved in DMSO. For example, the extract of isolate Streptomyces sp. strain Stup16_ B49.2 showed two distinct peaks in the HPLC chromatogram. The first peak had a retention time of $8.13 \mathrm{~min}$ and the second peak eluates at $13.24 \mathrm{~min}$ (Fig. 1). The main peak (retention time: $13.24 \mathrm{~min}$ ) showed antibacterial activity against $S$. aureus and E. coli. In general, all obtained fractions of each individual peak from the HPLC separation of the investigated extracts were analysed for inhibitory activity by measuring growth kinetics using the OmniLog instrument.

\section{Identification of the Extracted Compounds}

The active compounds from seven strains of Streptomyces species, named Stup16_B49.2, sima1_6, Stup16_B146, OPOK MB_B11,Stup18_J70,OPOK_MB_A9, W367A and one strain of Micromonospora sp. Stup16_C148, were characterised chemically. HPLC-purified organic fractions of these strains showing antibacterial properties were subjected to MALDI-TOF MS measurement for molecular mass determination followed by MALDI-TOF MS/MS to obtain their fragmentation pattern. Mass spectrometric data were acquired using an Autoflex Speed (Bruker Daltonics) equipped with a $355 \mathrm{~nm} \mathrm{Nd:YAG} \mathrm{laser.} \mathrm{During} \mathrm{the} \mathrm{measurement,}$ the instrument was operating in reflectron mode with positive polarity. The matrix used was 2,5-dihydroxybenzoic acid dissolved in pure methanol at concentration of $15 \mathrm{mg} / \mathrm{ml}$. HPLC purified fractions were also dissolved in methanol and mixed with matrix solution. To facilitate the ion formation the sample-matrix mixture was spiked with a solution of $\mathrm{NaCl}$ saturated in pure ethanol. Identical measuring conditions were applied to obtain the spectra of reference standards and isolated HPLC compounds. The software mMass (open source mass spectrometry tool) version 5.5.0 was used to create mass spectra plots from measured raw data.

Based on the measured mass the most similar compound was identified by comparison with following databases: Reaxys, StreptomeDB, NPEdia, SuperNaturalDatabase and the Natural Product Atlas. StreptomeDB for example contains more than 2,400 different secondary metabolites produced from more than 1,900 strains of Streptomyces. ${ }^{[15]}$ MS/MS fragmentation patterns were generated from commercially available standards and from the isolated compounds. The peak patterns of the acquired spectra were compared to determine whether the spectrum of the in- 
Fig. 1. HPLC chromatogram with isolated fractions of an ethyl acetate extract from Streptomyces sp. Stup16_B_49.2 grown in NL148sb medium. The main peak with a retention time of $13.24 \mathrm{~min}$ corresponds to echinomycin.

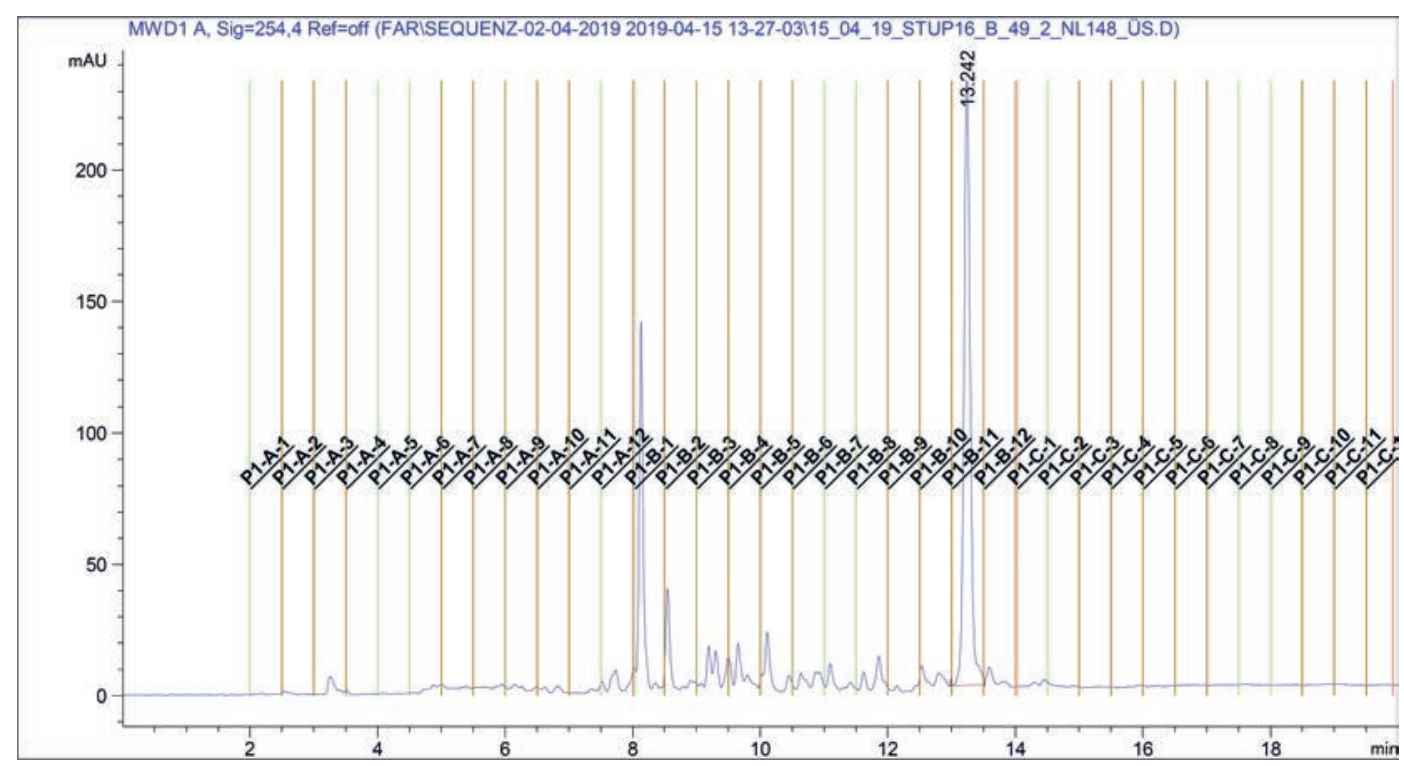

vestigated compound matches with the spectrum of the reference substance. Using this approach, we identified the antibiotic echinomycin from Streptomyces strains Stup16_B49.2 and sima 1_6. Echinomycin was detected as sodiated ion at $\mathrm{m} / \mathrm{z}$ of 1123.403 . The fragmentation patterns of echinomycin used as reference substance (CAS: 512-64-1, Sigma Aldrich) and the active substance isolated from Streptomyces strains Stup16_B49.2 generated with MALDI-TOF/TOF are shown in Fig. 2. The fragmentation patterns in the mass spectra of the isolated compound and the reference substance are identical.

The compound produced from Streptomyces strain Stup16_ B146 was able to inhibit $S$. aureus in the secondary screening using the OmniLog assay. The mass spectrometric analysis of this compound resulted in a mass-to-charge ratio $\mathrm{m} / \mathrm{z}$ peak at 825.522 . Comparing this mass with the NPEdia database TPU-0037-C (CAS: 485815-61-0, AdipoGen Life Sciences) was evaluated as a possible candidate for the reference standard. Although MS spectra of Stup16_B146 and TPU-0037-C were identical in terms of detected $\mathrm{m} / \mathrm{z}$ and isotopic pattern, the MS/MS fragmentation measurement did not result in superimposable spectra as shown in Fig. 3. The most intensive peaks of both spectra appeared at identical $\mathrm{m} / \mathrm{z}(70,156,227,296$ and 380), however several peaks were either only present in Stup16_B146 (m/z at 310, 394, 694) or had different relative intensity in comparison with TPU-0037-C

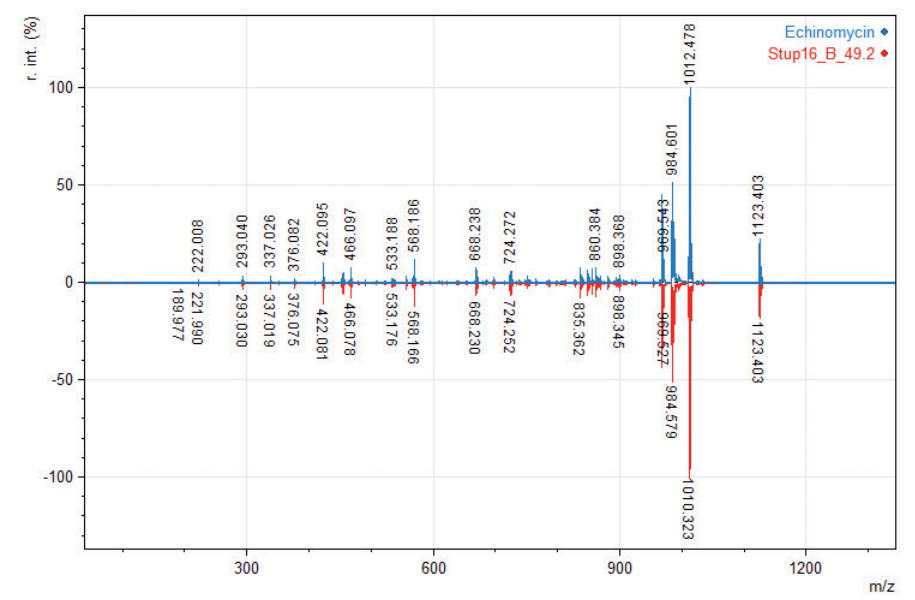

Fig. 2. MALDI-TOF MS/MS spectrum of the antimicrobial compound produced by Streptomyces sp. Stup16_B49.2 (red spectrum) is identical with the spectrum of reference substance echinomycin (blue spectrum). The molecular ion of echinomycin can be seen in the spectrum at $\mathrm{m} / \mathrm{z}$ 1123.403, which corresponds to the sodiated ion of the molecule. $(\mathrm{m} / \mathrm{z}$ at 85, 113, 156, 240 and 478). Following the identification scheme proposed by Schymanski et al. ${ }^{[16]}$ this result can be classified with confidence level 3 , which means tentative identification of a structure, where multiple structures are possible $(e . g$. positional isomers). Further structure elucidation of this compound is therefore needed. A natural compound produced from Streptomyces sp. strain OPOK_MB_B11 has $\mathrm{m} / \mathrm{z}$ of 1202.751 detected as sodiated ion and is proposed as azalomycin $\mathrm{F}_{4 \mathrm{a}}$ 2-ethylpentyl ester. However, a comparison of the fragmentation patterns is not possible since the substance is not commercially available. Streptomyces sp. strain W367A, isolated from the Cantone of Lucerne, produced a compound which was detected as sodiated ion at $\mathrm{m} / \mathrm{z}$ of 550.343. Comparing this mass with the StreptomeDB database, dihydropicromycin was identified as the most similar compound. One rare actinomycete strain, isolated from the soil of the riverbank of the Limmat in Unterengstringen, Canton of Zurich, was identified as Micromonospora and was able to inhibit $S$. aureus in the secondary screening. The compound of the isolated strain Micromonospora sp. Stup16_C148 has mass-to-charge ratio $\mathrm{m} / \mathrm{z}$ of 823.440 when detected as protonated ion. This matches with bottromycin A2, which has a monoisotopic neutral mass of 822.441 determined with high resolution electron impact mass spectrometry. ${ }^{[17]}$ Streptomyces sp. Stup18_J70 was isolated from forest soil of the Canton of Valais and showed strong inhibition of S. aureus in the screening assays. The isolated compound has a molecular weight of 787.449 and the obtained mass spectrum using MALDI-TOF MS is identical to a bafilomycin A1 derivative, which is described in the document CN104817547 (B) (patent in Chinese). The structures of the compounds from the investigated actinomycetes strains isolated in Switzerland are shown in Fig. 4.

\section{Streptomyces Strain Showing Cytotoxic Activity}

The in vitro cytotoxic activity of the Streptomyces sp. strain OPOK_MB_A9 was investigated using the human colon cancer HT- 29 cell line (DSMZ no.: ACC 299). The viability of the cells was measured using the MTT assay to estimate cell growth inhibition in the presence of the active compound of the HPLC fraction dissolved in DMSO. HT-29 cells were cultured in RPMI 1640 medium supplemented with $10 \%(v / v)$ fetal bovine serum, and $2 \mathrm{mM}$ L-glutamine and seeded into 96-well microtiter plates at a concentration of $10^{4}$ cells per well. Viable cells were able to convert the yellow MTT (tetrazolium salt) to purple-coloured formazan. The produced crystalline formazan was solubilised with DMSO after medium removal and a washing step with phosphate buffer saline. Optical densities (OD) were measured at $570 \mathrm{~nm}$ to de- 


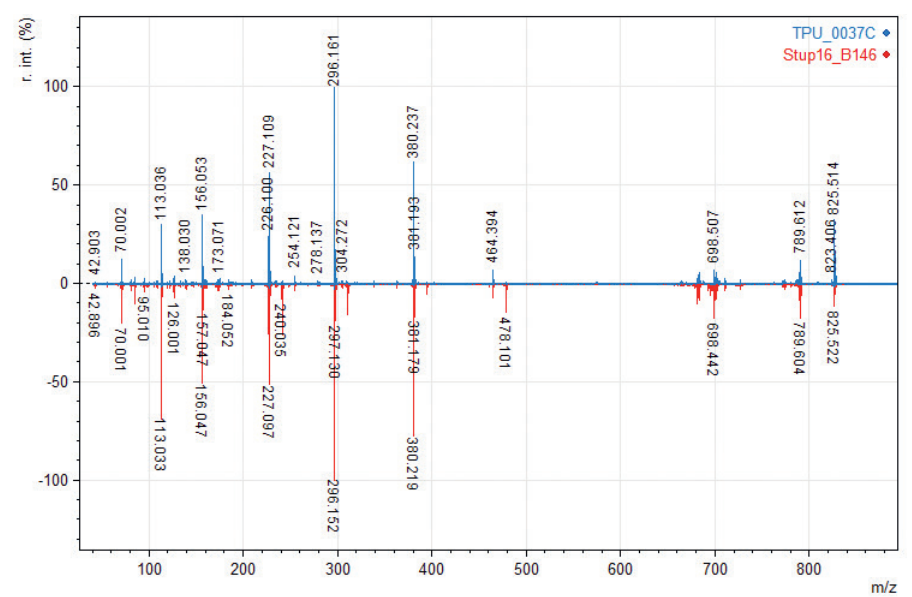

Fig. 3. MALDI-TOF MS/MS spectrum of the antimicrobial compound produced by Streptomyces sp. Stup16_B146 (red spectrum) and reference substance TPU-0037-C (blue spectrum).

termine the cytotoxicity effect of the fraction from Streptomyces sp. strain OPOK_MB_A9 on HT-29 cells. DMSO (1\% v/v) and ionomycin $(1 \% \mathrm{v} / \mathrm{v})$ were used as negative and positive control in the assay. The inhibition of the growth of HT-29 cells in the wells of the MTT assay was calculated according to the following equation:

$\%$ inhibition $=100-\left[\left(\mathrm{Abs}_{\text {sample }}-\mathrm{Abs}_{\text {blank }}\right) /\left(\mathrm{Abs}_{\text {control }}-\mathrm{Abs}_{\text {blank }}\right)\right] \times 100$

where $\mathrm{Abs}_{\text {sample }}$ is the absorbance of the compound in the HPLC fraction, $\mathrm{Abs}_{\text {blank }}$ is the absorbance of the medium in the well without HT-29 cells to measure the background and Abs is the absorbance of the control reaction, which contained all reagents except the compound.
The fraction of Streptomyces sp. strain OPOK MB_A9 exhibited a strong cytotoxic activity towards HT-29 cells causing about $75.8 \%$ inhibition after $24 \mathrm{~h}$ of incubation. DMSO showed no effect on the viability of the HT-29 cells and ionomycin caused $77.3 \%$ inhibition of the used human colon cancer cell line during the incubation time of $24 \mathrm{~h}$. Other extracts from actinomycetes strains tested were not able to reduce the viability of HT-29 cells. Ionomycin used as positive control in the MTT assay showed the same inhibitory effect on HT-29 cells as the tested fraction from Streptomyces sp. strain OPOK_MB_A9. The mass spectrum determined with MALDI-TOF MS confirmed that the molecular weight of the compound causing cytotoxic activity was 701.444 as a protonated ion. The obtained mass was compared with the used databases and matches with a macrolide antibiotic similar to milbemycin- $\alpha 8$.

\section{Genome Sequence Data from Actinokineospora Strain}

To assess the full potential of secondary metabolite production of investigated strains, genome mining is a valuable approach to identify biosynthetic gene clusters and to predict the bioactive compounds encoded from these groups of genes.

The genus Actinokineospora comprises spore-forming members, which form a most distant cluster in the suborder Pseudonocardineae. ${ }^{[27]}$ From the strain B136.1, isolated from grassland soil in the Canton of Lucerne, a partial 16S rRNA gene sequence of 1,295 bp amplified with PCR was determined and the BLASTn search indicate that the isolate belongs to the genus Actinokineospora. The 16S rRNA gene sequence of the strain has been deposited in the European Nucleotide Archive (ENA) and received LR740774 as accession number (study PRJEB35546). The draft genome sequence from Actinokineospora strain B136.1 was determined using $150 \mathrm{bp}$ paired-end Illumina MiSeq and nanopore-based genome sequencing. For genomic sequencing, the total DNA was prepared from $50 \mathrm{ml}$ of strain B136.1 culture

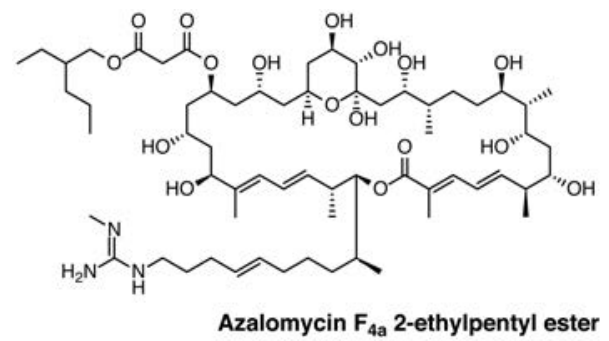

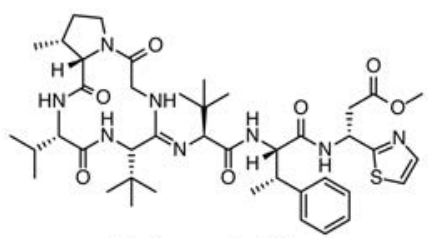

Bottromycin A2

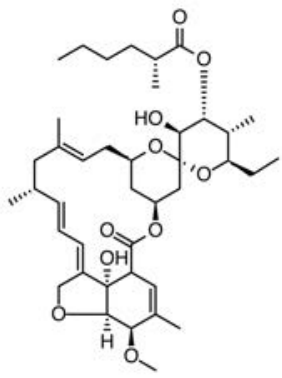

Milbemycin- $a 8$
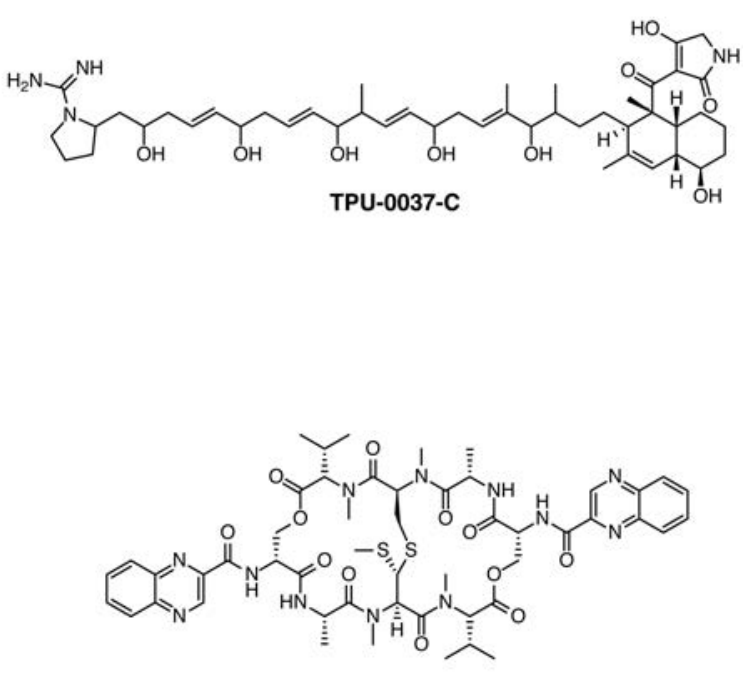

Echinomycin
Fig. 4. Structures of the identified antibiotic compounds from isolated actinomycetes strains in Switzerland, namely azalomycin $\mathrm{F}_{4 \mathrm{a}}$ 2-ethylpentyl ester, ${ }^{[18,19]} \mathrm{TPU}$ $0037-\mathrm{C},{ }^{[20,21]}$ bottromycin $\mathrm{A} 2,{ }^{[22]}$ echinomycin, ${ }^{[23]}$ milbemycin- $\alpha 8,{ }^{[24]}$ dihydropicromycin. ${ }^{[25]}$ The structure of bafilomycin A1 is shown in the publication of Yu et al. ${ }^{[26]}$ and a proposed derivative of the compound is published in the patent CN104817547 (B).

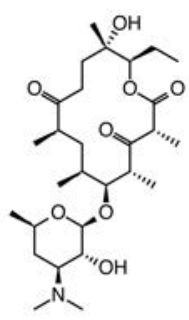

Dihydropicromycin 
grown in NL148sb medium at $28{ }^{\circ} \mathrm{C}$ on a rotary shaker at 120 rpm for several weeks up to one month. Cells were harvested, resuspended in $8 \mathrm{ml}$ of $10 \mathrm{mM}$ Tris $\mathrm{HCl} \mathrm{pH} \mathrm{8.0,1} \mathrm{mM} \mathrm{EDTA}$ and treated with $100 \mu \mathrm{g} / \mathrm{ml}$ RNAse A (DNAse free) and $40 \mathrm{mg} /$ $\mathrm{ml}$ lysozyme at $55^{\circ} \mathrm{C}$ for $30 \mathrm{~min}$. Two proteinase $\mathrm{K}$ incubation steps were performed. First, $8 \mathrm{ml}$ of $10 \mathrm{mg} / \mathrm{ml}$ proteinase $\mathrm{K}$ (in $2 \%$ SDS) was added. After $2 \mathrm{~h}$ of incubation at $55{ }^{\circ} \mathrm{C}, 100 \mu \mathrm{L}$ proteinase $\mathrm{K}(20 \mathrm{mg} / \mathrm{ml})$ was pipetted to the extraction procedure and the solution was incubated overnight. A phenol-chloroform extraction was performed, followed by DNA precipitation using ethanol and spectrophotometric quantification. ${ }^{[28]}$

The output of the hybrid assembly using Unicycler v.0.4.7 was 242 contigs with a size of about 7.6 Mbp. The complete $16 \mathrm{~S}$ rDNA sequence of $1,506 \mathrm{bp}$ was extracted from the genome and used for phylogenetic analysis. A phylogenetic tree was reconstructed to determine the taxonomic position and relationship of strain B136.1 to other species of Actinokineospora and closely related taxa. The evolutionary history of the tree was inferred by using the Maximum Likelihood method and Tamura-Nei model.[29] The evolutionary analyses were conducted in MEGA X. ${ }^{[30]}$ The $16 \mathrm{~S}$ rDNA sequence of strain B136.1 is identical to Actinokineospora sp. strain R19-10 (NBRC 105648) which was isolated from soil of Rishiri Island, Hokkaido, Japan. Based on the phylogenetic tree, both strains form a separate lineage of evolutionary divergence (Fig. 5). The separate lineage of both strains B136.1 and NBRC 105648 within the phylogenetic tree reconstructed indicate that both strains from Switzerland and Japan could be a new species within the genus Actinokineospora. The genetic relatedness between the two genomes was predicted using the average nucleotide identity (ANI) value based on MUMmer comparisons (ANIm) using pyANI v.0.2.4. Genomes of two strains from one species typically share $\geq$ 95\% ANI values among themselves. The boundary of DNA-DNA hybridization value of $70 \%$ for species delineation corresponds to 95\% ANI.[31] The calculated ANI values of strain B136.1 ranging from $85 \%$ to $86 \%$ with other species of the genus Actinokineospora support the evidence that the strain belongs to a new species.

The obtained genome sequence of Actinokineospora sp. B136.1 was analysed with the antiSMASH 3.0 program to determine the secondary metabolite gene clusters of the strain. More than 20 biosynthetic gene clusters including polyketide synthase (PKS-I and PKS-II) and nonribosomal peptide synthetases (NRPS) genes responsible for the biosynthetic pathways of the secondary metabolites have been detected. Based on the genome mining with antiSMASH, at least nine biosynthetic gene clusters were identified in strain B136.1 encoding for antibacterial properties. The following gene clusters with high and low identity values to known clusters encoding secondary metabolites with potential antibacterial activities were: actinokineosin $(90 \%$ identity with the most similar known cluster), friulimicin $(18 \%$ identity), malacidin (38\% identity), laspartomycin (4\% identity), abyssomicins M-X (62\% identity), indanomycin (26\% identity), hygromycin B (6\% identity), amycomicin (25\% identity), pristinamycin (29\% identity), respectively. In addition, strain B136.1 has a biosynthetic gene cluster, which is $20 \%$ identical to vicenistatin as most similar gene cluster. Vicenistatin, biosynthesised by the polyketide pathway, has been shown to possess antitumoral activity against human leukaemia and colon adenocarcinoma cells. ${ }^{[32]}$ None of the biosynthetic gene clusters identified on the genome of Actinokineospora sp. strain B136.1 were expressed under the cultivation conditions with the three different media (R2A, GYM4, NL148sb) used in the laboratory.

\section{Discussion}

The World Health Organisation warns that multidrug-resistant bacteria are currently one of the greatest threats to public health and immediate action is urgently needed to increase investments in the discovery of new medicines. ${ }^{[33]}$ Many strains of
Actinobacteria have already been screened for antibiotic production using traditional screening procedures leading to the identification of known and new antibacterial compounds. The large diversity of actinomycetes strains in the environment represents a unique source for detection and isolation of natural compounds with antibacterial activities. In this work, we have focused on the isolation of actinomycetes strains from Switzerland and the identification of antibiotics produced by the investigated strains. Different types of sampling sites were selected to increase the biodiversity of isolated strains.

Echinomycin and TPU-0037-C are available as reference standards allowing the generation and comparison of fragmentation patterns in MALDITOF/TOF mass spectrometry. Compounds similar to azalomycin $\mathrm{F}_{4 \mathrm{a}}$ 2-ethylpentyl ester, milbemycin- $\alpha 8$, a derivative of bafilomycin A1, dihydropicromycin and bottromycin A2 have been detected from different Streptomyces strains and one Micromonospora isolate based on their mass spectra.

Echinomycin is active against clinical isolates of $S$. aureus and is more potent in comparison to vancomycin. ${ }^{[34]}$ Echinomycin belongs to the quinomycin class peptide antibiotics containing two quinoxaline-2-carbonyl chromophores important for interaction with DNA. QXC (quinoxaline-2-carboxylic acid) is the aromatic precursor of echinomycin and is produced from the amino acid L-tryptophan. At the beginning of the QXC biosynthesis, L-tryptophan is loaded with the help of a small $\mathrm{MbtH}$-like protein onto a non-ribosomal peptide synthetase which has both adenylation and peptidyl carrier protein domains. The genes responsible for the biosynthesis of QXC have been detected in Streptomyces griseovariabilis subsp. bandungensis showing homology to the echinomycin $(\mathrm{ecm})$ and triostin A (trs) gene clusters from S. lasaliensis and S. triostinicus, respectively. ${ }^{\left[{ }^{[3]}\right]}$ Echinomycin is not a rare antibiotic and was repeatedly detected in this study using HPLC and MALDI-TOF/TOF analysis. Combining genome mining in identifying genes involved in secondary metabolite production and chemical biology using analytical assays will help to identify natural compounds, especially in cases where a standard for comparison of the detected compound is not available.

Compounds related to TPU-0037-C and azalomycin $\mathrm{F}_{4 \mathrm{a}}$ 2-ethylpentyl ester have been detected in this study from two Streptomyces isolates. TPU-0037-C produced from Streptomyces platensis strain TP-A0598 is a structural analogue to lydicamycin and has been shown to inhibit different Gram-positive bacteria including methicillin-resistant $S$. aureus but failed to inhibit Gramnegative bacteria like E. coli. ${ }^{[20]}$ Azalomycin F4a 2-ethylpentyl ester is a new macrocyclic lactone produced from a Streptomyces sp. strain 211726 isolated from mangrove soil and exhibited antifungal activity against Candida albicans and moderate cytotoxicity against human colon cancer cell line HCT-116. ${ }^{[18]}$ Azalomycin F showed inhibition against Bacillus subtilis and was not able to inhibit $E$. coli even at higher concentrations. ${ }^{[36]}$ Bottromycin A2 was identified from the isolated Micromonospora strain Stup16_C148. Bottromycin A2 inhibits bacterial protein synthesis and is active against clinical isolated strains of methicillin-resistant $S$. aureus and vancomycin-resistant enterococci. ${ }^{[37]}$ Bottromycin was first isolated from the fermentation broth of Streptomyces bottropensis by Waisvisz et al. ${ }^{[38]}$ and the antibiotic is also produced by Micromonospora chalcea. ${ }^{[39]}$ The structure of bottromycin A2 is unique in nature due to the macrocyclic amidine, rare $\beta$-methylated amino acids residues and a terminal thiazole. ${ }^{[40]}$ Bottromycin A2 is not in clinical use since the antibiotic is unstable in vivo. ${ }^{[37]}$ Old antibiotics could undergo a renaissance by modifying them chemically to generate more stable and powerful drugs to treat diseases caused by pathogenic bacteria.

Most of the antibiotics used today originated from actinomycetes with Streptomyces being the main producer. For example, daunorubicin and doxorubicin produced by Streptomyces peucetius are two anthracycline antibiotics and both are used in human 


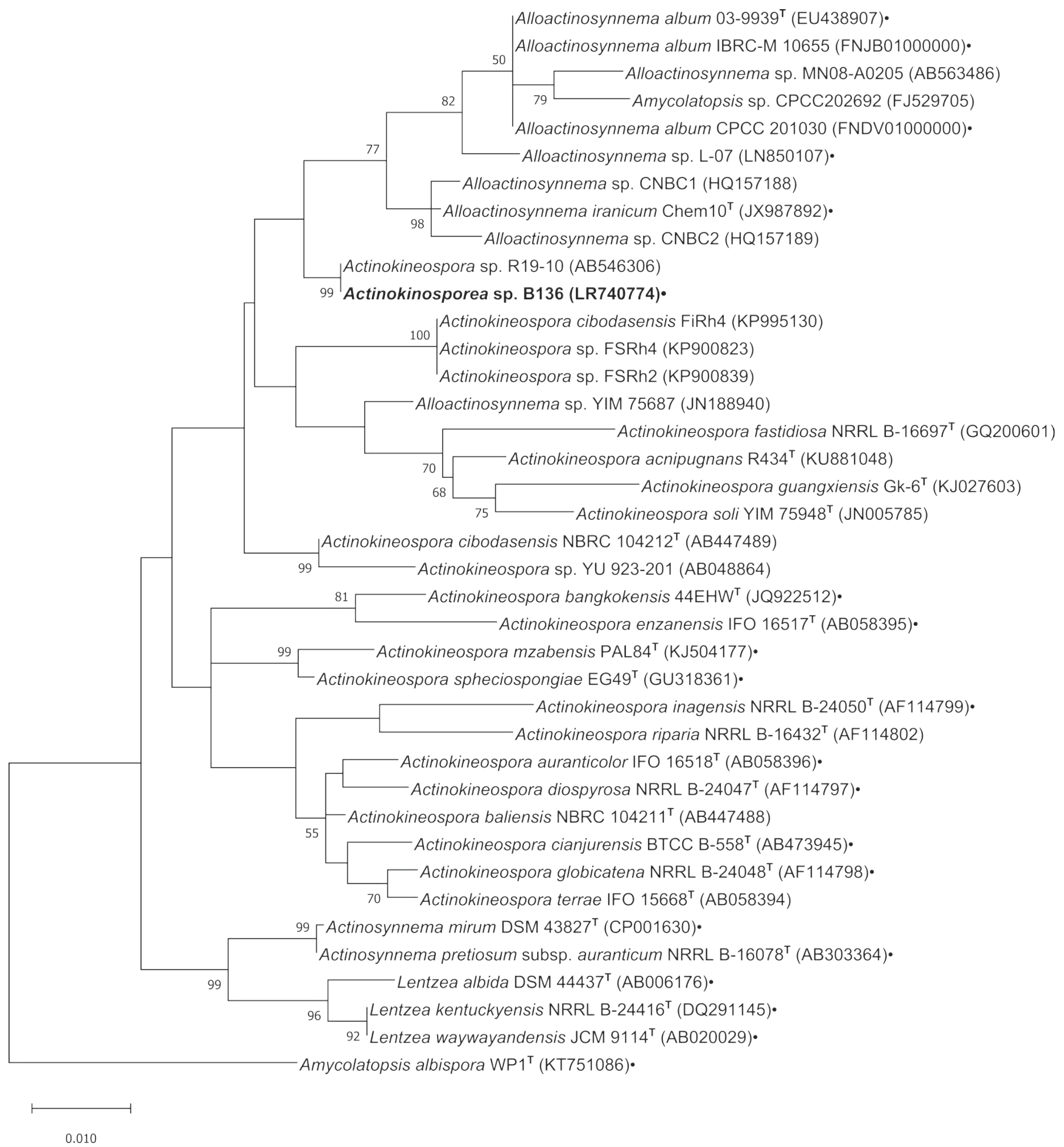

Fig. 5. Phylogenetic position of Actinokineospora B136.1 with its closely related taxa based on 16S rRNA sequences. The tree with the highest log likelihood (-5946.57) is shown. The percentage of trees out of 1000 replications in which the associated taxa clustered together is shown next to the branches when above $50 \%$, and the scale bar represents the percentage of substitutions per site. Initial tree(s) for the heuristic search were obtained automatically by applying Neighbor-Joining and BioNJ algorithms to a matrix of pairwise distances estimated using the Maximum Composite Likelihood (MCL) approach, and then selecting the topology with superior log likelihood value. The type strain of the species is highlighted with a bold superscript T after the strain information; accession numbers are provided between brackets after the strain information and genome sequenced strains are highlighted with a dot after the accession number. Amycolatopsis albispora WP1 ${ }^{\top}$ was used as outgroup.

cancer chemotherapy. ${ }^{[41]}$ Actinomycetes are well known for their production of natural compounds with different biological activities, including antitumoral properties. Important compounds used in chemotherapy to treat cancer are of microbial origin. Many antitumoral compounds produced by actinomycetes have in addition also antimicrobial activity. Streptomyces sp. OPOK_MB_A9 produced a compound similar to milbemycin- $\alpha 8$ and was found to possess inhibitory properties against colon adenocarcinoma cell line HT-29. Milbemycins are macrolide antibiotics and were isolated from the mycelium of Streptomyces hygroscopicus subsp. aureolacrimosus obtained from a fermentation broth, which showed activity against Acarus and larvae of the insects of the or- 
der Lepidoptera. ${ }^{[42]}$ Milbemycin derivatives were able to inhibit $\mathrm{ABC}$ transporter, an efflux pump involved in azole resistance, in Candida glabrata and C. albicans. ${ }^{[43]}$ Drug resistance is a natural phenomenon that hampers not only the performance of antibiotics. It also impedes drugs for other major clinical indications, such as antifungals as well as compounds for the treatment of cancer, viral infections, or parasitic diseases. ${ }^{[44]}$ Microbial extract screening from actinomycetes isolates could yield very potent natural products to fight this challenge. For example, milbemycin compounds are promising agents to overcome multidrug resistance in cancer chemotherapy. ${ }^{[45]}$ Streptomyces sp. strain Stup18_J70 produced a compound similar to bafilomycin A1. Bafilomycins are macrolide antibiotics with a 16-membered lactone ring and were first isolated from Streptomyces griseus strains. ${ }^{[46]}$ The most intensively studied bafilomycin is bafilomycin A1, which specifically inhibits vacuolar $\mathrm{H}^{+}$-ATPases as shown with vacuolar membranes derived from a fungus (Neurospora crassa), a plant (Zea mays) and an animal (bovine chromaffin granule membranes from the adrenal medulla). ${ }^{[4]}$ Due to targeting V-ATPases, bafilomycin A1 prevents the acidification of autophagosomes, leading to disruption of the formation of autolysosomes and inhibition of endogenous protein degradation affecting autophagy. ${ }^{[48]}$

Several isolated strains in this study from different species of actinomycetes, mainly rare actinomycetes, were not able to produce secondary metabolites under the used standard cultivation conditions. The determined genome sequence of Aktinokineospora sp. strain B136.1 revealed that the strain is able to produce several natural compounds but failed to produce them under different media tested. It has been reported that Streptomyces coelicolor and S. griseus, for example, possess more than twenty different biosynthetic gene clusters encoding for secondary metabolites but produce only few compounds in culture. ${ }^{[49]}$ The challenge therefore is to find ways to activate the transcription of silent biosynthetic gene clusters in actinomycetes to isolate novel compounds. The «one strain many compounds» (OSMAC) approach refers to the ability of one strain to produce several compounds when cultivated under different cultivation conditions. ${ }^{[50]}$ It is postulated that chemical compounds like rare earth elements or $\mathrm{N}$-acetylglucosamine present in the environment but not found in culture medium triggers secondary metabolite production of Streptomyces. ${ }^{[51]}$ One successful strategy to awaken secondary metabolite production is the cocultivation of strains of Streptomyces with mycolic acid-containing bacteria. Eight novel 5-alkyl-1,2,3,4-tetrahydroquinolines showing antifungal activity against yeast were detected by cocultivation of Streptomyces nigrescens HEK616 and Tsukamurella pulmonis P-B0596.[52] Streptomyces endus S522 produced alchivemycin A in combined culture with T. pulmonis, which is an antibiotic with a novel heterocyclic ring structure exhibiting antimicrobial activity at low concentrations against Micrococcus luteus. ${ }^{[53]}$ Quorum sensing is involved in the regulation of secondary metabolites in Actinobacteria and the signalling molecule $\gamma$-butyrolactone (GBL) seems to be limited to Actinobacteria. ${ }^{[5]}$ Autoinducer molecules like $\gamma$-butyrolactones are strongly associated in Streptomyces with the regulation of antibiotic production and morphological differentiation. The autoregulatory factor induces streptomycin production and sporulation in Streptomyces griseus. ${ }^{[54]}$ Treatment of strains with quorum sensing molecules could trigger new metabolite production. The addition of $\gamma$-butyrolactone SCB1 in culture of S. coelicolor A3(2) induces the production of actinorhodin and undecylprodigiosin, which are two pigmented antibiotics. ${ }^{[55]}$

Genome editing strategies offer opportunities to engineer strains for biotechnological applications. An exciting application is to use CRISPR/Cas technology to alter target sequences in the genome of Streptomyces. A CRISPR/Cas9 system was developed to mutate the beta-ketoacyl synthase subunit beta of the blue pig- ment actionorhodin (act) gene cluster in S. coelicolor M145. The wild type $S$. coelicolor strain M145 has a blue colour appearance based on actinorhodin pigment production. The mutated strain M145/pWHU2659 ( $\triangle$ actI-ORF2) showed an orange colour from undecylprodigiosin visible after loss of the blue pigment actinorhodin. ${ }^{[56]}$ CRISPR/Cas is used to activate silent biosynthetic gene clusters, which are not expressed under standard cultivation conditions, to find new natural products. A novel compound was detected by an engineered Streptomyces viridochromogenes strain DSM 40736 by insertion of constitutive promoter kasOp* constructed by Wang et al..$^{[57]}$ in front of the biosynthetic operon SSQG_RS26895-26920 of an uncharacterized PKS type II gene cluster. The engineered $S$. viridochromogenes strain produced a brown pigment before sporulation, which is a novel polyketide with a dihydrobenzo[ $\alpha]$ naphthacenequinone core. ${ }^{[58]}$ Studies to activate silent biosynthetic gene clusters in the genome of actinomycetes strains with this technique are currently performed in our laboratory.

Beside the production of natural drugs, Streptomyces has additional properties producing enzymes, which are important biocatalysts like halogenase, polyhydroxyalkanoate depolymerase, cytochrome P450 monooxygenase, methyl transferase and imine reductase. ${ }^{[59]}$ The degradation of staurosporine to the less toxic aglycon K-252c using Streptomyces mediocidicus ATCC 13279 has been described by Ghisalba and coworkers. ${ }^{[60]}$ Identifying microbial strains with interesting and novel properties facilitate their use in further innovative applications. Access to high quality biological resources and associated data is important to foster research and application in biotechnology. To support the life science community with well-characterised strains, Prof. Dr. Oreste Ghisalba encouraged deposits of microbial resources for long-term maintenance in open collections in accordance with the Nagoya Protocol in order to make them available for research activities in academia, research institutions and industry. Preservation of interesting strains is of great importance to prevent loss of microbial resources and to utilise them for further applications in the future. ${ }^{[61]}$ The published actinomycetes strains isolated in Switzerland will be deposited in the Culture Collection of Switzerland (CCOS). The CCOS was established in 2009 as the national culture collection of Switzerland based on a national need and initiative of Prof. Dr. Oreste Ghisalba. He would certainly enjoy the structural beauty and diversity of the natural substances with antibiotic effects to be discovered from actinomycetes strains.

\section{Acknowledgements}

The authors would like to thank Tobias Wermelinger, Dennis Wipfli, Marina Starc and our apprenticeship students for their help with some of the experiments as well as the department's HPC team for the compute resources and support. The project was supported by the Department of Life Sciences and Facility Management of the Zurich University of Applied Sciences (ZHAW) in Wädenswil, Switzerland and is part of the Health Research Hub to promote interdisciplinary research in the field of health.

Received: February 24, 2020

[1] M. F. Chellat, L. Raguž, R. Riedl, Angew. Chem. Int. Ed. 2016, 55, 6600, doi: 10.1002/anie.201506818.

[2] M. F. Chellat, R. Riedl, Angew. Chem. Int. Ed. 2017, 56, 13184, doi: 10.1002/ anie. 201708133.

[3] A. van der Meij, S. F. Worsley, M. I. Hutchings, G. P. van Wezel, FEMS Microbiol. 2017, 41, 392, doi: 10.1093/femsre/fux005.

[4] J. Bobek, K. Smídová, M. Čihák, Front Microbiol. 2017, 8, 2205. doi: 10.3389/fmicb.2017.02205.

[5] A. V. Polkade, S. S. Mantri, U. J. Patwekar, K. Jangid, Front. Microbiol. 2016, 7, 131. doi: $10.3389 /$ fmicb.2016.00131.

[6] H. B. Woodruff, Appl. Environ. Microbiol. 2014, 80, 2, doi: 10.1128/ AEM.01143-13.

[7] K. Lewis, Nat. Rev. Drug. Discov. 2013, 12, 371, doi: 10.1038/nrd3975. 
[8] G. B. Mahajan, L. Balachandran, Front. Biosci. (Elite Ed). 2012, 4, 240. doi: $10.2741 / 373$

[9] E. Gavrish, C. S. Sit, S. Cao, O. Kandror, A. Spoering, A. Peoples, L. Ling, A. Fetterman, D. Hughes, A. Bissell, H. Torrey, T. Akopian, A. Mueller, S Epstein, A. Goldberg, J. Clardy, K. Lewis. Chem. Biol. 2014, 21, 509, doi: 10.1016/j.chembiol.2014.01.014.

[10] A. L. Kunz, A. Labes 1, J. Wiese, T. Bruhn, G. Bringmann, J. F. Imhoff, Mar Drugs 2014, 12, 1699, doi: 10.3390/md12041699.

[11] E. B. Shirling, D. Gottlieb, Int. J. Syst. Bacteriol. 1966, 16, 313, doi: 10.1099/00207713-16-3-313.

[12] J. Zhang, L. Zhang, Mod. Appl. Sci. 2011, 5, 124, doi: 10.5539/mas v5n2p124.

[13] V. Cordovez, V. J. Carrion, D. W. Etalo, R. Mumm, H. Zhu, G. P. van Wezel, J. M. Raaijmakers, Front. Microbiol. 2015, 6, 1081, doi: 10.3389/ fmicb.2015.01081.

[14] L. M. Schmitz, J. Schäper, K. Rosenthal, S. Lütz, ChemCatChem. 2019, 11, 5766, doi: 10.1002/cctc.201901273.

[15] X. Lucas, C. Senger, A. Erxleben, B. A. Grüning, K. Döring, J. Mosch S. Flemming, S. Günther, Nucleic Acids Res. 2013, 41, doi: 10.1093/nar/ gks1253.

[16] E. L. Schymanski, J. Jeon, R. Gulde, K. Fenner, M. Ruff, H. P. Singer, J. Hollender, Environ. Sci. Technol. 2014, 48, 2097, doi: 10.1021/es5002105.

[17] Y. Takahashi, H. Naganawa, T. Takita, H. Umezawa, S. Nakamura, J. Antibiot. (Tokyo), 1976, 29, 1120, doi: 10.7164/antibiotics.29.1120.

[18] G. J. Yuan, K. Hong, H. P. Lin, J. Li, Chin. Chem. Lett. 2010, 21, 947, doi: 10.1016/j.cclet.2010.03.025.

[19] G. Yuan, K. Hong, H. Lin, Z. She, J. Li, Mar. Drugs 2013, 11, 817, doi $10.3390 / \mathrm{md} 11030817$.

[20] T. Furumai, K. Eto, T. Sasaki, H. Higuchi, H. Onaka, N. Saito, T. Fujita, H. Naoki, Y. Igarashi, J. Antibiot (Tokyo) 2002, 55, 873, doi: 10.7164/antibiotics.55.873.

[21] Y. Hayakawa, N. Kanamaru, N. Morisaki, H. Seto, Tetrahedron Lett. 1991 , $32,2,213$.

[22] H. Shimamura, H. Gouda, K. Nagai, T. Hirose, M. Ichioka, Y. Furuya, Y Kobayashi, S. Hirono, T. Sunazuka, S. Omura, Angew. Chem. Int. Ed. 2009, 48, 914, doi: 10.1002/anie.200804138.

[23] M. Sato, T. Nakazawa, Y. Tsunematsu, K. Hotta, K. Watanabe, Curr. Opin. Chem. Biol. 2013, 17, 537, doi: 10.1016/j.cbpa.2013.06.022.

[24] S. E. Blanchflower, R. J. J. Dorgan, J. R. Everett, S. A. Readshaw, Tetrahedron Lett. 1988, 29, 6645, doi: 10.1016/S0040-4039(00)82419-3.

[25] J. Majer, J. B. McAlpine, R. S. Egan, J. W. Corcoran, J. Antibiot. (Tokyo) 1976, 29, 769, doi:10.7164/antibiotics.29.769.

[26] Z. Yu, L.-X. Zhao, C.-L. Jiang, Y. Duan, L. Wong, K. C. Carver, L. A. Schuler, B. Shen, J. Antibiot. (Tokyo) 2011, 64, 159, doi: 10.1038/ja.2010.147.

[27] D. P. Labeda, M. Goodfellow, J. Chun, X.-Y. Zhi, W.-J. Li, Int. J. Syst. Evol. Microbiol. 2011, 61, 1259, doi: 10.1099/ijs.0.024984-0.

[28] P. Pujic, A. Bolotin, P. Fournier, A. Sorokin, A. Lapidus, K. H. Richau, J. Briolay, F. Mebarki, P. Normand, A. Sellstedt, Genome Announc. 2015, 3 , doi: 10.1128/genomeA.01387-15.

[29] K. Tamura, M. Nei, Mol. Biol. Evol. 1993, 10, 512, doi: 10.1093/oxfordjournals.molbev.a040023

[30] S. Kumar, G. Stecher, M. Li, C. Knyaz, K. Tamura, Mol. Biol. Evol. 2018, 35, 1547, doi: 10.1093/molbev/msy096.

[31] J. Goris, K. T. Konstantinidis, J. A. Klappenbach, T. Coenye, P. Vandamme, J. M. Tiedje, Int. J. Syst. Evol. Microbiol. 2007, 57, 81, doi: 10.1099/ ijs.0.64483-0.

[32] K. Shindo, M. Kamishohara, A. Odagawa, M. Matsuoka, H. Kawai, $J$. Antibiot. (Tokyo). 1993, 46, 1076, doi: 10.7164/antibiotics.46.1076.

[33] WHO, 2015, ISBN 9789241509763.

[34] Y.-S. Park, W.-S. Shin, S.-K. Kim, J. Antimicrob. Chemother. 2008, 61, 163 , doi: $10.1093 / \mathrm{jac} / \mathrm{dkm} 421$.

[35] C. Zhang, L. Kong, Q. Liu, X. Lei, T. Zhu, J. Yin, B. Lin, Z. Deng, D. You, PloS One 2013, 8, doi: 10.1371/journal.pone.0056772.

[36] S. Sugawara, J. Antibiot. (Tokyo) 1968, 21, 83, doi: 10.7164/antibiotics.21.83.
[37] Y. Kobayashi, M. Ichioka, T. Hirose, K. Nagai, A. Matsumoto, H. Matsui, H. Hanaki, R. Masuma, Y. Takahashi, S. Omura, T. Sunazuka. Bioorg. Med. Chem. Lett. 2010, 20, 6116, doi: 10.1016/j.bmcl.2010.08.037.

[38] J. M. Waisvisz, M. G. van der Hoeven, J. van Peppen, W. C. M. Zwennis, J. Am. Chem. Soc. 1957, 79, 4520, doi: 10.1021/ja01573a072.

[39] G. H. Wagman, M. J. Weinstein, Ann. Rev. Microbiol. 1980, 34, 537, doi: 10.1146/annurev.mi.34.100180.002541

[40] W. J. K. Crone, N. M. Vior, J. Santos-Aberturas, L. G. Schmitz, F. J. Leeper, A. W. Truman, Angew. Chem. Int. Ed. 2016. 55, 9639, doi: 10.1002/ anie. 201604304

[41] G. Aubel-Sadron, D. Londos-Gagliardi, Biochimie 1984, 66, 333, doi:10.1016/0300-9084(84)90018-x.

[42] Y. Takiguchi, H. Mishima, M. Okuda, M. Terao, A. Aoki, R. Fukuda, J. Antibiot. (Tokyo) 1980, 33, 1120, doi: 10.7164/antibiotics.33.1120.

[43] L. V. Silva, M. Sanguinetti, P. Vandeputte, R. Torelli, B. Rochat, D. Sanglard, Antimicrob. Agents Chemother. 2013, 57, 873, doi: 10.1128/AAC.02040-12.

[44] S. Agnello, M. Brand, M. F. Chellat, S. Gazzola, R. Riedl, Angew. Chem. Int. Ed. 2019, 58, 3300, doi: 10.1002/anie.201802416.

[45] A. Gao, H. Liang, X. Wang, X. Zhang, M. Jing, J. Zhang, Y. Yan, W. Xiang, Eur. J. Pharmacol. 2011, 659, 108, doi: 10.1016/j.ejphar.2011.03.023.

[46] G. Werner, H. Hagenmaier, H. Drautz, A. Baumgartner, H. Zähner, J. Antibiot. (Tokyo) 1984, 37, 110, doi: 10.7164/antibiotics.37.110.

[47] E. J. Bowman, A. Siebers, K. Altendorf, Proc. Natl. Acad. Sci. USA 1988, 85, 7972, 10.1073/pnas.85.21.7972.

[48] A. Yamamoto, Y. Tagawa, T. Yoshimori, Y. Moriyama, R. Masaki, Y. Tashiro, Cell Struct. Funct. 1998, 23, 33, doi: 10.1247/csf.23.33.

[49] Y. Tanaka, K. Kasahara, Y. Hirose, K. Murakami, R. Kugimiya, K. Ochi, J. Bacteriol. 2013, 195, 2959, doi: 10.1128/JB.00147-13.

[50] H. B. Bode, B. Bethe, R. Höfs, A. Zeeck, ChemBioChem. 2002, 3, 619, doi: 10.1002/1439-7633(20020703)3:7<619::AID-CBIC619>3.0.CO;2-9.

[51] S. Antoraz, R.I. Santamaría, M. Díaz, D. Sanz, H. Rodríguez, Front Microbiol. 2015, 13, 461. doi: 10.3389/fmicb.2015.00461.

[52] R. Sugiyama, S. Nishimura, T. Ozaki, S. Asamizu, H. Onaka, H. Kakeya, Org. Lett. 2015. 17, 1918. doi: 10.1021/acs.orglett.5b00607.

[53] H. Onaka, J. Antibiot. (Tokyo) 2017, 70, 865, doi: 10.1038/ja.2017.51.

[54] E. Takano, Curr. Opin. Microbiol. 2006, 9, 287, doi: 10.1016/j. mib.2006.04.003.

[55] E. Takano, T. Nihira, Y. Hara, J. J. Jones, C. J. L. Gershater, Y. Yamada, M. Bibb, J. Biol. Chem. 2000, 275, 11010, doi: 10.1074/jbc.275.15.11010.

[56] H. Zeng, S. Wen, W. Xu, Z. He, G. Zhai, Y. Liu, Z. Deng, Y. Sun, Appl. Microbiol. Biotechnol. 2015, 99, 10575, doi: 10.1007/s00253-015-6931-4.

[57] W. Wang, X. Li, J. Wang, S. Xiang, X. Feng, K. Yang, Appl. Environ. Microbiol. 2013, 79, 4484, doi: 10.1128/AEM.00985-13.

[58] M. M. Zhang, F. T. Wong, Y. Wang, S. Luo, Y. H. Lim, E. Heng, W. L. Yeo, R. E. Cobb, B. Enghiad, E. L. Ang, H. Zhao, Nat. Chem. Biol. 2017, doi: 10.1038/nchembio.2341.

[59] J. Spasic, M. Mandic, L. Djokic, J. Nikodinovic-Runic, Appl. Microbiol. Biotechnol. 2018, 102, 3513, doi: 10.1007/s00253-018-8884-x.

[60] K. Goeke, P. Hoehn, O. Ghisalba, J. Antibiot. (Tokyo) 1995, 48, 428, doi: 10.7164/antibiotics.48.428.

[61] M. Sievers, in 'Encyclopedia of Metagenomics', Ed. K. Nelson, New York, 2013, pp 111, doi: 10.1007/978-1-4614-6418-1_741-4.

\section{License and Terms}

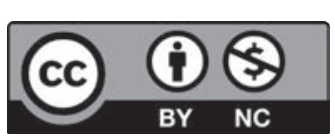

This is an Open Access article under the terms of the Creative Commons Attribution License CC BY_NC 4.0. The material may not be used for commercial purposes.

The license is subject to the CHIMIA terms and conditions: (http:// chimia.ch/component/sppagebuilder/?view = page \&id=12).

The definitive version of this article is the electronic one that can be found at doi:10.2533/chimia.2020.382 